Published in final edited form as:

Atherosclerosis. 2009 October ; 206(2): 575-580. doi:10.1016/j.atherosclerosis.2009.03.032.

\title{
Association of serum myeloperoxidase with the ankle-brachial index and peripheral arterial disease
}

\author{
Zeenat Ali, \\ Division of Cardiovascular Diseases, Mayo Clinic \\ Paul Sarcia, \\ Department of Internal Medicine, Mayo Clinic \\ Thomas H Mosley Jr, \\ Department of Medicine (Geriatrics), University of Mississippi Medical Center \\ Venkateswarlu Kondragunta, and \\ Division of Cardiovascular Diseases, Mayo Clinic \\ Iftikhar J Kullo \\ Division of Cardiovascular Diseases, Mayo Clinic
}

\section{Abstract}

\begin{abstract}
Myeloperoxidase (MPO) is an enzymatic mediator of several inflammatory cascades and higher serum levels have been associated with increased risk of adverse cardiovascular events. We investigated the association of serum MPO with the ankle-brachial index (ABI) and peripheral arterial disease (PAD) in a bi-ethnic cohort of African-Americans and non-Hispanic white individuals. Participants included 1324 African-Americans (mean age 64 years, 71\% women) and 1237 non-Hispanic white individuals (mean age 59 years, $57 \%$ women) belonging to hypertensive sibships. Serum levels of MPO were measured by solid phase sandwich immunoassay. ABI was measured using a standard protocol and PAD was defined as an ABI $<0.90$. Multivariable regression analysis using generalized estimating equations were performed to assess whether serum MPO levels were associated with ABI and the presence of PAD. After adjustment for age and sex, higher MPO levels were significantly associated with lower ABI and the presence of PAD in African-Americans ( $p=0.004$ and $p=0.005$, respectively) and in non-Hispanic white individuals ( $p=0.001$ and $p=0.016$, respectively). After additional adjustment for conventional risk factors (diabetes, smoking status, total and high-density lipoprotein cholesterol, waist circumference, hypertension), prior history of myocardial infarction or stroke, and medication use (statins, aspirin, estrogen), higher MPO levels remained significantly associated with lower ABI and the presence of PAD in both African-Americans ( $p=0.008$ and $p=0.010$, respectively) and non-Hispanic white individuals ( $p=0.001$ and $p=0.018$, respectively). We conclude that higher MPO levels are associated with lower ABI and the presence of PAD in African-Americans and non-Hispanic white individuals.
\end{abstract}

\section{Keywords \\ ankle-brachial index; inflammation; myeloperoxidase; peripheral arterial disease}

\author{
(c) The Author(s), 2009. \\ Corresponding author: Iftikhar J Kullo, Division of Cardiovascular Diseases, Mayo Clinic, 200 First Street Southwest, Rochester, MN \\ 55905, USA. kullo.iftikhar@mayo.edu. \\ Disclosures \\ There are no conflicts of interest to disclose.
}




\section{Introduction}

Inflammation plays a key role in the development and evolution of atherosclerotic plaque. ${ }^{1}$ Myeloperoxidase (MPO), an enzyme secreted by activated neutrophils, participates in various inflammatory processes involved in atherosclerosis. ${ }^{2,3}$ MPO has been implicated in the initiation and progression of atherosclerotic lesions through mechanisms related to its role in low-density lipoprotein (LDL) oxidation, ${ }^{2}$ consumption of nitric oxide leading to endothelial dysfunction ${ }^{4,5}$ and generation of numerous oxidative reactants and diffusible free radical species. ${ }^{6}$ Using immunohistochemistry and mass spectrometry, atherosclerotic plaques have been shown to be abundant in oxidation products generated by MPO.,7-9 MPO levels are associated with the risk of developing coronary artery disease in apparently healthy individuals, ${ }^{10}$ with the presence and severity of coronary artery disease, ${ }^{11,12}$ and are predictive of adverse outcomes in patients presenting with chest pain ${ }^{13}$ or an acute coronary syndrome. ${ }^{14}$

Peripheral arterial disease (PAD) is a manifestation of atherosclerosis and affects at least eight million people in the USA. ${ }^{15,16}$ Prior reports indicate that inflammation plays an important role in the development, ${ }^{17}$ progression ${ }^{18}$ and severity ${ }^{19}$ of PAD. Whether MPO is associated with systemic atherosclerotic burden is not known. We therefore investigated whether serum MPO is associated with the ankle-brachial index (ABI) and the presence of $\mathrm{PAD}$, as defined by $\mathrm{ABI}<0.90$. Furthermore, we investigated whether any association is independent of plasma C-reactive protein (CRP), a marker of systemic inflammation.

\section{Methods}

The study was part of the Proteomic Markers of Arteriosclerosis Study which is investigating the association of markers in various etiologic pathways of vascular disease with several phenotypes of arteriosclerosis. ${ }^{20}$ Participants were from the Genetic Epidemiology Network of Arteriopathy (GENOA) study, a multicenter, community-based study that aims to identify genetic variants influencing blood pressure (BP) levels and the development of target-organ damage due to hypertension. ${ }^{21}$ Participants were enrolled if two or more members of a sibship had hypertension before the age of 60 years. If the eligible proband had at least one sibling with hypertension, all available full biologic siblings of the index hypertensive siblings were invited to participate in the study. The only exclusionary criterion at enrollment was the presence of a secondary cause of hypertension (such as documented renal artery stenosis or advanced renal insufficiency) in the index sibs.

The study was approved by the Institutional Review Boards of the University of Mississippi Medical Center; Jackson, MS and the Mayo Clinic, Rochester, MN. Written informed consent was obtained from each participant. The present study included 1324 AfricanAmericans and 1237 non-Hispanic white individuals.

Height measured by stadiometer and weight measured by electronic balance were used to calculate body mass index (BMI; $\mathrm{kg} / \mathrm{m}^{2}$ ). The diagnosis of hypertension was based on participants reporting a prior diagnosis of hypertension and current use of prescription antihypertensive medications, or systolic blood pressure (SBP) of $140 \mathrm{mmHg}$ or higher or diastolic blood pressure (DBP) of $90 \mathrm{mmHg}$ or higher measured at the study visit. Diabetes was considered present if the participant was being treated with insulin or oral hypoglycemic agents or had a fasting glucose level $\geq 126 \mathrm{mg} / \mathrm{dl}$. Ever-smoking was defined as having smoked > 100 cigarettes. Information about the use of medications was obtained from the participants at the time of the study visit. 
Blood was drawn by venipuncture after an overnight fast. Serum total cholesterol and highdensity lipoprotein (HDL) cholesterol were measured by standard enzymatic methods.

\section{Serum MPO and plasma CRP assays}

Serum MPO levels were measured by a solid phase sandwich ELISA (ALPCO Diagnostics, Salem, $\mathrm{NH}$ ). Intra-assay coefficients of variation (CVs) were $7.4 \%$ and $7.4 \%$ at 4.1 and $165.6 \mathrm{ng} / \mathrm{ml}$, respectively, and inter-assay CVs were $10.7 \%$ and $12.4 \%$ at 2.8 and $52.2 \mathrm{ng} /$ $\mathrm{ml}$, respectively. Plasma CRP levels were measured by a highly sensitive immunoturbidimetric assay. Inter-assay CVs were: $14 \%, 3.2 \%, 3.4 \%$, and $3.6 \%$ at 0.33 , $1.05,9.07$, and $23.8 \mathrm{mg} / \mathrm{dl}$, respectively.

\section{Ankle-brachial index $(\mathrm{ABI})$}

At each center, examiners who underwent training in Mayo Clinic's non-invasive vascular laboratory in Rochester, MN measured the ABI. This identical, standardized protocol was used at both centers. ABI was measured as follows: After a 5-minute rest, participants were evaluated in the supine position. Appropriately sized BP cuffs were placed on each arm and ankle, and a Doppler ultrasonic instrument (Medisonics; Minneapolis, MN, USA) was used to detect arterial signals. The cuff was inflated to $10 \mathrm{mmHg}$ above SBP and deflated at 2 $\mathrm{mmHg} / \mathrm{s}$. The first reappearance of the arterial signal was taken as the SBP. To calculate the $\mathrm{ABI}$, the SBP at each ankle site (posterior tibial and dorsalis pedis arteries) was divided by the higher of the two brachial pressures. The lower of the average ABIs from the two legs was used in the analyses. ABI $<0.90$ or $>1.4$ was used to indicate the presence of PAD. Participants with an ABI > $1.4(n=28)$ may have non-compressible arteries due to medial arterial calcification, ${ }^{22}$ and were excluded in the linear regression models but included in the logistic regression models as having PAD.

\section{Statistical methods}

Continuous data were summarized as either mean $\pm \mathrm{SD}$ or median and quartiles and categorical data were expressed as percentages (\%). Owing to significant differences in age and the proportion of women in the two ethnic groups, differences in characteristics between the two ethnic groups were assessed after adjustment for age and sex. Serum MPO and plasma CRP were log transformed to reduce skewness. Spearman correlations between serum MPO and plasma CRP were calculated in each ethnic group.

Analyses to assess whether serum MPO is independently associated with ABI and PAD were performed separately in each ethnic group. In each ethnic group, multiple regression models were constructed to include age, sex, waist circumference (as a measure of adiposity), history of smoking, diabetes, hypertension, total cholesterol, HDL cholesterol, medication (statin, aspirin, estrogen) use, and previous history of myocardial infarction or stroke. Backward elimination was performed to identify variables independently associated with ABI. Age and sex were forced into all multivariable regression models. Because of the presence of sibships in the sample, regression analyses were performed using generalized estimating equations (GEE) ${ }^{23}$ We also assessed whether MPO contributed to the prediction of presence of PAD. Models before and after the addition of MPO were compared by calculating the c-statistic from receiver operating characteristic (ROC) curve analyses.

A two-sided $p$-value of $<0.05$ was deemed statistically significant. Statistical analyses were carried out using the SAS v 8.2 (SAS Institute, Cary, NC, USA) software package. 


\section{Results}

Table 1 shows baseline characteristics of the two ethnic groups. African-Americans were significantly older and had higher risk factor burden compared to non-Hispanic white individuals. The proportion of women was higher in both ethnic groups and the majority of participants were hypertensive. Use of statins, estrogen and aspirin was less frequent in African-Americans than their non-Hispanic white counterparts. ABI was lower in AfricanAmericans as compared to non-Hispanic white individuals $(p<0.001)$. Serum MPO levels were higher in African-Americans than in non-Hispanic white individuals $(p<0.0001)$. MPO levels were significantly correlated with CRP levels in African-Americans $(\rho=0.260$, $p<0.0001)$ and a weaker correlation was noted in non-Hispanic white individuals $(\rho=$ $0.128, p<0.0001)$.

In African-Americans, after adjustment for age and sex in linear regression models, higher MPO levels were significantly associated with a lower ABI $(p=0.004)$. The association remained significant after further adjustment for conventional risk factors $(p=0.008)$. However, after additional adjustment for plasma CRP, the association of higher MPO levels with lower ABI was of borderline significance $(p=0.094)$ (Table 2$)$. The results of multivariable logistic regression models were consistent with those of linear regression models. After adjustment for age and sex, higher MPO levels were significantly associated with presence of $\operatorname{PAD}(p=0.005)$. After additional adjustment for conventional risk factors, higher MPO levels remained significantly associated with the presence of PAD $(p=0.01)$. However, the association was not statistically significant after additional adjustment for plasma CRP ( $p=0.193)$ (Table 3). In ROC curve analysis, the c-statistic for predicting PAD was 0.784 using age, sex and conventional risk factors. After addition of MPO, the cstatistic was essentially unchanged (0.785).

In non-Hispanic white individuals, after adjustment for age and sex in linear regression models, higher MPO levels were significantly associated with lower ABI $(p=0.001)$. The association was robust to additional adjustment for conventional risk factors $(p=0.001)$ and plasma CRP ( $p=0.001$ ) (Table 2). The results of multivariable logistic regression models were consistent with those of linear regression models. After adjustment for age and sex, higher MPO levels were significantly associated with the presence of PAD $(p=0.016)$. The association remained significant after further adjustment for conventional risk factors $(p=$ $0.018)$ and plasma CRP $(p=0.022)$ (Table 3$)$. In ROC curve analysis, the c-statistic for predicting PAD was 0.856 using age, sex and conventional risk factors. After addition of MPO, the c-statistic increased modestly to 0.866 .

\section{Discussion}

The results of this study demonstrate that higher serum MPO levels are associated with lower $\mathrm{ABI}$ and presence of PAD, independent of age, sex, and conventional risk factors. Additional adjustment for plasma CRP, a marker of systemic inflammation, attenuated these associations in African-Americans but not in non-Hispanic whites. The addition of MPO to a model including age, sex, and conventional risk factors, led to a modest increase in the cstatistic for the presence of PAD in non-Hispanic white individuals but not in AfricanAmericans.

The results of several recent studies indicate that MPO plays a key role in the evolution of atherosclerosis, through various mechanisms leading to initiation, propagation and subsequent complications of atherosclerotic plaque formation. MPO-generated reactive species promote oxidation, ${ }^{24,25}$ lipid peroxidation ${ }^{26}$ and crosslinking of LDL, ${ }^{27}$ facilitating uptake by macrophages. ${ }^{2,28}$ MPO also plays a role in the generation of dysfunctional 
HDL $^{29-31}$ and interferes with its capacity to promote cholesterol efflux. ${ }^{32}$ MPO leads to endothelial dysfunction by serving as an enzymatic sink for nitric oxide, limiting its bioavailability and function. ${ }^{33-35}$ MPO-derived reactive species contribute to plaque destabilization and rupture by activating various protease cascades that affect the stability and thrombogenicity of plaques. ${ }^{36-38}$

Several studies have shown an association between circulating levels of MPO and coronary heart disease. In a prospective, nested, case-control study conducted among apparently healthy men and women ( $n=1138$ for cases and $n=2237$ for controls), baseline MPO levels were significantly higher in individuals who developed coronary heart disease during 8 years of follow-up than among those who did not. ${ }^{10}$ Higher serum MPO levels have been associated with the presence ${ }^{11}$ and severity ${ }^{12}$ of coronary artery disease on angiography and are predictive of adverse outcomes in patients presenting to an emergency department with chest pain. ${ }^{13}$ In the CAPTURE study (a randomized placebo-controlled trial of abciximab before and during coronary intervention in refractory unstable angina, $n=1090$ ), serum MPO was an independent predictor of adverse cardiac outcomes over 6 months of followup. ${ }^{14}$ Brevetti et al. ${ }^{39}$ demonstrated that in patients with PAD $(n=156)$, higher serum MPO levels were associated with increased risk of developing myocardial infarction/ stroke.

To the best of our knowledge, the present study is the first to show a cross-sectional association between serum levels of MPO and presence of PAD. Adjustment for conventional risk factors did not attenuate the association, indicating that serum MPO is not strongly associated with such risk factors. Indeed, we confirmed that serum MPO was poorly correlated with age, sex, presence of hypertension/diabetes, plasma lipids and smoking status (analyses not shown). Thus, MPO appears to be an 'orthogonal' marker that may have clinical utility in assessing the risk for developing PAD. In ROC curve analyses, there was no increase in the c-statistic for predicting PAD after the addition of MPO to a model that included age, sex, and conventional risk factors in African-Americans and only a small increase in c-statistic in non-Hispanic white individuals. Single markers may not yield a significant increase in the c-statistic and multiple markers may be needed to obtain predictive information that is incremental to conventional risk factors. ${ }^{40,41}$ Further investigation is needed to assess the utility of MPO in multimarker panels for predicting the risk of PAD. ${ }^{42}$

A strength of the present study is the inclusion of a large bi-ethnic cohort of individuals from the community. We used uniform protocols in the two ethnic groups including questionnaires, anthropometric measurements, assessment of conventional risk factors, and the MPO assay. Limitations include the cross-sectional nature of the study, making it difficult to make inferences about causality. Confounding by unknown potential causal factors cannot be ruled out. The majority of the study participants were hypertensive and, therefore, the findings may not be generalizable to the entire population and further studies need to be done in normotensives and younger adults.

In conclusion, our study demonstrates an association between serum MPO levels and PAD, a surrogate for systemic atherosclerosis, independent of conventional risk factors. However, the association, although statistically significant, was modest and not independent of plasma CRP in African-Americans. Further investigation is needed to assess whether MPO could serve as a biomarker for future risk of PAD and to identify PAD patients at risk for adverse outcomes.

\section{Acknowledgments}

The authors would like to acknowledge Guanghui Liu for help with statistical analyses. This work was funded by grants HL81331 and HL75794 from the National Institutes of Health. 


\section{References}

1. Ross R. Atherosclerosis — an inflammatory disease. N Engl J Med. 1999; 340:115-126. [PubMed: 9887164]

2. Podrez EA, Schmitt D, Hoff HF, Hazen SL. Myeloperoxidase-generated reactive nitrogen species convert LDL into an atherogenic form in vitro. J Clin Invest. 1999; 103:1547-1560. [PubMed: 10359564]

3. Sugiyama S, Okada Y, Sukhova GK, Virmani R, Heinecke JW, Libby P. Macrophage myeloperoxidase regulation by granulocyte macrophage colony-stimulating factor in human atherosclerosis and implications in acute coronary syndromes. Am J Pathol. 2001; 158:879-891. [PubMed: 11238037]

4. Eiserich JP, Baldus S, Brennan ML, et al. Myeloperoxidase, a leukocyte-derived vascular NO oxidase. Science. 2002; 296:2391-2394. [PubMed: 12089442]

5. Vita JA, Brennan ML, Gokce N, et al. Serum myeloperoxidase levels independently predict endothelial dysfunction in humans. Circulation. 2004; 110:1134-1139. [PubMed: 15326065]

6. Klebanoff SJ. Oxygen metabolism and the toxic properties of phagocytes. Ann Intern Med. 1980; 93:480-489. [PubMed: 6254418]

7. Heinecke JW. Mass spectrometric quantification of amino acid oxidation products in proteins: insights into pathways that promote LDL oxidation in the human artery wall. Faseb J. 1999; 13:1113-1120. [PubMed: 10385603]

8. Podrez EA, Abu Soud HM, Hazen SL. Myeloperoxidase-generated oxidants and atherosclerosis. Free Radic Biol Med. 2000; 28:1717-1725. [PubMed: 10946213]

9. Daugherty A, Dunn JL, Rateri DL, Heinecke JW. Myeloperoxidase, a catalyst for lipoprotein oxidation, is expressed in human atherosclerotic lesions. J Clin Invest. 1994; 94:437-444. [PubMed: 8040285]

10. Meuwese MC, Stroes ES, Hazen SL, et al. Serum myeloperoxidase levels are associated with the future risk of coronary artery disease in apparently healthy individuals: the EPIC-Norfolk Prospective Population Study. J Am Coll Cardiol. 2007; 50:159-165. [PubMed: 17616301]

11. Zhang R, Brennan ML, Fu X, et al. Association between myeloperoxidase levels and risk of coronary artery disease. JAMA. 2001; 286:2136-2142. [PubMed: 11694155]

12. Buffon A, Biasucci LM, Liuzzo G, D'Onofrio G, Crea F, Maseri A. Widespread coronary inflammation in unstable angina. N Engl J Med. 2002; 347:5-12. [PubMed: 12097534]

13. Brennan M-L, Penn MS, Van Lente F, et al. Prognostic value of myeloperoxidase in patients with chest pain. N Engl J Med. 2003; 349:1595-1604. [PubMed: 14573731]

14. Baldus S, Heeschen C, Meinertz T, et al. Myeloperoxidase serum levels predict risk in patients with acute coronary syndromes. Circulation. 2003; 108:1440-1445. [PubMed: 12952835]

15. Regensteiner JG, Hiatt WR. Current medical therapies for patients with peripheral arterial disease: a critical review. Am J Med. 2002; 112:49-57. [PubMed: 11812407]

16. Hirsch AT, Haskal ZJ, Hertzer NR, et al. ACC/AHA Guidelines for the management of patients with peripheral arterial disease (lower extremity, renal, mesenteric, and abdominal aortic). Circulation. 2006; 113:e463-e654. [PubMed: 16549646]

17. Wildman RP, Muntner P, Chen J, Sutton Tyrrell K, He J. Relation of inflammation to peripheral arterial disease in the national health and nutrition examination survey, 1999-2002. Am J Cardiol. 2005; 96:1579-1583. [PubMed: 16310445]

18. Tzoulaki I, Murray GD, Lee AJ, Rumley A, Lowe GD, Fowkes FG. C-reactive protein, interleukin-6, and soluble adhesion molecules as predictors of progressive peripheral atherosclerosis in the general population: Edinburgh Artery Study. Circulation. 2005; 112:976983. [PubMed: 16087797]

19. Silvestro A, Scopacasa F, Ruocco A, et al. Inflammatory status and endothelial function in asymptomatic and symptomatic peripheral arterial disease. Vasc Med. 2003; 8:225-232. [PubMed: 15125481]

20. Gerszten RE, Accurso FJ, Bernard GR, et al. Challenges in translating plasma proteomics from bench to bedside: update from the NHLBI Clinical Proteomics Programs. Am J Physiol Lung Cell Mol Physiol. 2008; 295:L16-L22. [PubMed: 18456800] 
21. Boerwinkle E. Multi-center genetic study of hypertension: The Family Blood Pressure Program (FBPP). Hypertension. 2002; 39:3-9. [PubMed: 11799070]

22. Hiatt WR. Medical treatment of peripheral arterial disease and claudication. N Engl J Med. 2001; 344:1608-1621. [PubMed: 11372014]

23. Zeger SL, Liang KY. Longitudinal data analysis for discrete and continuous outcomes. Biometrics. 1986; 42:121-130. [PubMed: 3719049]

24. Leeuwenburgh C, Rasmussen JE, Hsu FF, Mueller DM, Pennathur S, Heinecke JW. Mass spectrometric quantification of markers for protein oxidation by tyrosyl radical, copper, and hydroxyl radical in low density lipoprotein isolated from human atherosclerotic plaques. J Biol Chem. 1997; 272:3520-3526. [PubMed: 9013599]

25. Leeuwenburgh C, Hardy MM, Hazen SL, et al. Reactive nitrogen intermediates promote low density lipoprotein oxidation in human atherosclerotic intima. J Biol Chem. 1997; 272:1433-1436. [PubMed: 8999808]

26. Hazen SL, Zhang R, Shen Z, et al. Formation of nitric oxide-derived oxidants by myeloperoxidase in monocytes: pathways for monocyte-mediated protein nitration and lipid peroxidation in vivo. Circ Res. 1999; 85:950-958. [PubMed: 10559142]

27. Heinecke JW, Li W, Francis GA, Goldstein JA. Tyrosyl radical generated by myeloperoxidase catalyzes the oxidative cross-linking of proteins. J Clin Invest. 1993; 91:2866-2872. [PubMed: 8390491]

28. Hazell LJ, Stocker R. Oxidation of low-density lipoprotein with hypochlorite causes transformation of the lipoprotein into a high-uptake form for macrophages. Biochem J. 1993; 290:165-172. [PubMed: 8439285]

29. Zheng L, Nukuna B, Brennan ML, et al. Apolipoprotein AI is a selective target for myeloperoxidase-catalyzed oxidation and functional impairment in subjects with cardiovascular disease. J Clin Invest. 2004; 114:529-541. [PubMed: 15314690]

30. Zheng L, Settle M, Brubaker G, et al. Localization of nitration and chlorination sites on apolipoprotein A-I catalyzed by myeloperoxidase in human atheroma and associated oxidative impairment in ABCA1-dependent cholesterol efflux from macrophages. J Biol Chem. 2005; 280:38-47. [PubMed: 15498770]

31. Bergt C, Pennathur S, Fu X, et al. The myeloperoxidase product hypochlorous acid oxidizes HDL in the human artery wall and impairs ABCA1-dependent cholesterol transport. Proc Natl Acad Sci U S A. 2004; 101:13,032-13,037.

32. Marsche G, Hammer A, Oskolkova O, Kozarsky KF, Sattler W, Malle E. Hypochlorite-modified high density lipoprotein, a high affinity ligand to scavenger receptor class B, type I, impairs high density lipoprotein-dependent selective lipid uptake and reverse cholesterol transport. J Biol Chem. 2002; 277:32,172-32,179. [PubMed: 11602593]

33. Harrison DG. Cellular and molecular mechanisms of endothelial cell dysfunction. J Clin Invest. 1997; 100:2153-2157. [PubMed: 9410891]

34. Forgione MA, Leopold JA, Loscalzo J. Roles of endothelial dysfunction in coronary artery disease. Curr Opin Cardiol. 2000; 15:409-415. [PubMed: 11198623]

35. Abu, Soud HM.; Hazen, SL. Nitric oxide is a physiological substrate for mammalian peroxidases. J Biol Chem. 2000; 275:37,524-37,532.

36. Peppin GJ, Weiss SJ. Activation of the endogenous metalloproteinase, gelatinase, by triggered human neutrophils. Proc Natl Acad Sci U S A. 1986; 83:4322-4326. [PubMed: 3012563]

37. Shabani F, McNeil J, Tippett L. The oxidative inactivation of tissue inhibitor of metalloproteinase-1 (TIMP-1) by hypochlorous acid (HOCI) is suppressed by antirheumatic drugs. Free Radic Res. 1998; 28:115-123. [PubMed: 9645388]

38. Fu X, Kassim SY, Parks WC, Heinecke JW. Hypochlorous acid oxygenates the cysteine switch domain of promatrilysin (MMP-7). A mechanism for matrix metalloproteinase activation and atherosclerotic plaque rupture by myeloperoxidase. J Biol Chem. 2001; 276:41,279-41,287.

39. Brevetti G, Schiano V, Laurenzano E, et al. Myeloperoxidase, but not C-reactive protein, predicts cardiovascular risk in peripheral arterial disease. Eur Heart J. 2008; 29:224-230. [PubMed: 18156137] 
40. Wang TJ, Gona P, Larson MG, et al. Multiple biomarkers for the prediction of first major cardiovascular events and death. N Engl J Med. 2006; 355:2631-2639. [PubMed: 17182988]

41. Zethelius B, Berglund L, Sundstrom J, et al. Use of multiple biomarkers to improve the prediction of death from cardiovascular causes. N Engl J Med. 2008; 358:2107-2116. [PubMed: 18480203]

42. Fung ET, Wilson AM, Zhang F, et al. A biomarker panel for peripheral arterial disease. Vasc Med. 2008; 13:217-224. [PubMed: 18687758] 
Table 1

Participant characteristics $(n=2561)$

\begin{tabular}{|c|c|c|c|c|c|c|}
\hline & \multicolumn{3}{|c|}{ African-Americans $(n=1324)$} & \multicolumn{3}{|c|}{ Non-Hispanic white individuals $(n=1237)$} \\
\hline & $\begin{array}{c}\text { ABI }<0.9 \\
(n=151)\end{array}$ & $\begin{array}{l}\text { ABI } \geq 0.9 \\
(n=1143)\end{array}$ & $p$ & $\begin{array}{c}\text { ABI }<0.9 \\
(n=55)\end{array}$ & $\begin{array}{l}\text { ABI } \geq 0.9 \\
(n=1122)\end{array}$ & $p$ \\
\hline Age, years & $69.4 \pm 8.5$ & $62.7 \pm 9.1$ & $<0.0001$ & $67.7 \pm 8.1$ & $58.0 \pm 9.9$ & $<0.0001$ \\
\hline Men, $n(\%)$ & $55(36.4)$ & $320(28.0)$ & 0.032 & $24(43.6)$ & $486(43.3)$ & 0.963 \\
\hline BMI, $\mathrm{kg} / \mathrm{m}^{2}$ & $30.5 \pm 6.9$ & $31.5 \pm 6.4$ & 0.059 & $29.4 \pm 6.9$ & $30.9 \pm 6.3$ & 0.092 \\
\hline Waist circumference, $\mathrm{cm}$ & $104.2 \pm 13.7$ & $103.2 \pm 14.3$ & 0.395 & $100.2 \pm 14.8$ & $100.8 \pm 15.9$ & 0.789 \\
\hline Total cholesterol, mg/dl & $214.5 \pm 45.1$ & $200.4 \pm 40.5$ & 0.0001 & $188.9 \pm 36.0$ & $197.1 \pm 34.3$ & 0.086 \\
\hline HDL cholesterol, mg/dl & $55.2 \pm 16.7$ & $58.0 \pm 18.5$ & 0.078 & $49.7 \pm 16.3$ & $51.8 \pm 15.0$ & 0.306 \\
\hline Systolic BP, mmHg & $145.1 \pm 25.9$ & $137.7 \pm 20.1$ & $<0.0001$ & $138.0 \pm 19.8$ & $130.4 \pm 16.5$ & 0.001 \\
\hline Diastolic BP, mmHg & $76.2 \pm 12.2$ & $79.5 \pm 10.5$ & 0.0004 & $70.6 \pm 10.1$ & $74.1 \pm 9.0$ & 0.005 \\
\hline $\begin{array}{l}\text { Previous history of MI or } \\
\text { stroke }\end{array}$ & $25(16.6)$ & $95(8.3)$ & 0.001 & $12(21.8)$ & $73(6.5)$ & $<0.0001$ \\
\hline Smoking, $n(\%)$ & $86(57.0)$ & $436(38.1)$ & $<0.0001$ & $38(69.1)$ & $545(48.6)$ & 0.003 \\
\hline Diabetes, $n(\%)$ & $63(41.7)$ & $316(27.6)$ & 0.0004 & $17(30.9)$ & $153(13.6)$ & 0.0004 \\
\hline Hypertension, $n(\%)$ & $143(94.7)$ & 888 (77.7) & $<0.0001$ & $52(94.5)$ & $798(71.1)$ & 0.0002 \\
\hline Statin use, $n(\%)$ & $36(23.8)$ & $202(17.7)$ & 0.066 & $25(45.5)$ & 321 (28.6) & 0.007 \\
\hline Aspirin, $n(\%)$ & $63(41.7)$ & $362(31.7)$ & 0.014 & $34(61.8)$ & $452(40.3)$ & 0.002 \\
\hline Estrogen, $n(\%)$ & $24(15.9)$ & $195(17.1)$ & 0.719 & $4(7.3)$ & $263(23.4)$ & 0.005 \\
\hline $\mathrm{MPO}^{a}, \mathrm{ng} / \mathrm{ml}$ & $45.4 \pm 29.4$ & $42.8 \pm 29.4$ & 0.325 & $53.7 \pm 44.1$ & $34.2 \pm 28.9$ & $<0.0001$ \\
\hline $\mathrm{ABI}^{a, b}$ & $0.7 \pm 0.2$ & $1.0 \pm 0.1$ & $<0.0001$ & $0.8 \pm 0.1$ & $1.2 \pm 0.1$ & $<0.0001$ \\
\hline C-reactive protein, $\mathrm{mg} / \mathrm{dl}$ & $8.1 \pm 8.0$ & $5.5 \pm 6.1$ & $<0.0001$ & $4.8 \pm 6.5$ & $4.2 \pm 5.0$ & 0.372 \\
\hline
\end{tabular}

Data are presented as mean \pm SD or percentage of study participants.

BMI, body mass index; HDL, high-density lipoprotein; MPO, myeloperoxidase; ABI, ankle-brachial index.

In African-Americans, $n$ for MPO and ABI is 1164 and 1294, respectively; in Non-Hispanic white individuals, $n$ for MPO and ABI is 1215 and 1177 , respectively.

${ }^{b}$

$\mathrm{ABI}$ was missing in 30 African-Americans and 60 non-Hispanic white individuals. 


\section{Table 2}

Association of serum myeloperoxidase (MPO) with ankle-brachial index (ABI): linear regression models

\begin{tabular}{ccccccc}
\hline & \multicolumn{2}{c}{ African-Americans } & & \multicolumn{2}{c}{ Non-Hispanic white individuals } \\
\cline { 2 - 3 } & $\boldsymbol{\beta} \pm \mathbf{S E}$ & $\boldsymbol{p}$ & & $\boldsymbol{\beta} \pm \mathbf{S E}$ & $\boldsymbol{p}$ \\
\hline Model 1 & $-0.014 \pm 0.006$ & 0.019 & & $-0.021 \pm 0.006$ & 0.0001 \\
Model 2 & $-0.017 \pm 0.006$ & 0.004 & $-0.017 \pm 0.005$ & 0.001 \\
Model 3 & $-0.015 \pm 0.006$ & 0.008 & $-0.018 \pm 0.005$ & 0.001 \\
Model 4 & $-0.010 \pm 0.006$ & 0.094 & $-0.017 \pm 0.005$ & 0.001 \\
\hline
\end{tabular}

Model 1: Unadjusted.

Model 2: Adjusted for age and sex.

Model 3: Adjusted for age, sex, history of myocardial infarction or stroke, smoking, diabetes, hypertension, estrogen use, waist circumference, total and HDL cholesterol, and statin use.

Model 4: Adjusted for Model 3 variables and C-reactive protein. 


\section{Table 3}

Association of serum myeloperoxidase (MPO) with peripheral arterial disease (PAD): logistic regression models

\begin{tabular}{ccccccccc}
\hline & \multicolumn{3}{c}{ African-Americans } & & \multicolumn{2}{c}{ Non-Hispanic white individuals } \\
\cline { 2 - 3 } & Odds ratio & $\mathbf{9 5 \%} \mathbf{~ C I}$ & $\boldsymbol{p}$ & & Odds ratio & $\mathbf{9 5 \%} \mathbf{C I}$ & $\boldsymbol{p}$ \\
\hline Model 1 & 1.15 & $0.99-1.34$ & 0.066 & & 1.64 & $1.19-2.25$ & 0.002 \\
Model 2 & 1.26 & $1.07-1.48$ & 0.005 & 1.48 & $1.08-2.05$ & 0.016 \\
Model 3 & 1.24 & $1.05-1.47$ & 0.010 & 1.54 & $1.08-2.19$ & 0.018 \\
Model 4 & 1.12 & $0.94-1.34$ & 0.193 & 1.52 & $1.06-2.18$ & 0.022 \\
\hline
\end{tabular}

Odds ratios are shown for 1 standard deviation increase in log MPO.

PAD was defined as $\mathrm{ABI}<0.9$ or $>1.4$.

Model 1: Unadjusted.

Model 2: Adjusted for age and sex.

Model 3: Adjusted for age, sex, smoking, diabetes, hypertension, estrogen use, waist circumference, total and HDL cholesterol, and statin use.

Model 4: Adjusted for Model 3 variables and C-reactive protein. 